\title{
Patrones de evolución de filiales en entornos volátiles
}

\author{
Sigala Paparella, Luis E.* \\ León Darder, Fidel ${ }^{* *}$
}

\section{Resumen}

En este trabajo se aplican y se extienden los patrones de evolución de filiales planteados por Birkinshaw y Hood, al estudiar la influencia de los entornos volátiles sobre los procesos de evolución de las filiales de empresas multinacionales. Utilizando una metodología cualitativa a través de 41 estudios de casos de filiales de diversos sectores localizadas en Venezuela, se observó que las mismas siguen procesos conducentes a la mejora, el refuerzo y al declive de sus roles. En los resultados se identifican a la matriz y la propia filial como impulsores de esos procesos, pero se aprecia también al entorno local como determinante de la evolución de las filiales, con lo cual se extienden los patrones que plantean Birkinshaw y Hood que consideran sólo a la matriz y a la propia filial. Como conclusión, no sólo se resalta la importancia del entorno en la determinación del rol de las filiales, sino que se evidencia que los entornos volátiles pueden tener un impacto tanto positivo como negativo en la evolución de la filial.

Palabras clave: Filiales venezolanas, entorno volátil, empresas multinacionales, evolución de la filial.

\section{Recibido: 20-01-09. Aceptado: 15-05-10}

* Doctor en Dirección de Empresas, Universitat de València. Profesor Asociado, Decanato de Administración y Contaduría, Universidad Centroccidental "Lisandro Alvarado". Apartado postal 400. Barquisimeto, Venezuela. Isigala@ucla.edu.ve. Tel: +58 251 2591419, Fax: +58 251 2591461.

** Doctor en Ciencias Económicas, Universitat de València. Profesor Titular de Escuela Universitaria, Departamento de Dirección de Empresas “Juan José Renau Piqueras”, Facultat d'Economía, Universitat de València, España. Fidel.Leon@uv.es. 


\title{
Subsidiary Evolution Patterns in Volatile Environments
}

\begin{abstract}
This paper applies and extends Birkinshaw and Hood's evolution patterns for subsidiaries in a study of the influence of volatile environments on the evolutionary processes of multinational subsidiary corporations. Using qualitative methodology based on 41 study cases of subsidiaries from diverse sectors located in Venezuela, it was observed that the subsidiaries follow processes that lead to the improvement, reinforcement and the decline of their roles. Results identify the headquarters and the subsidiary itself as drivers for those processes, but they also appreciate the local environment as a determinant in subsidiary evolution, thereby extending the patterns Birkinshaw and Hood have proposed, which consider only the headquarters and the subsidiary. Conclusions highlight not only the importance of the environment in determining the role of the subsidiaries, but also the evidence that volatile environments can have a positive as well as a negative impact on subsidiary evolution.
\end{abstract}

Key words: Venezuelan subsidiaries, volatile environment, multinational corporations, subsidiary evolution.

\section{Introducción}

Las filiales exteriores ${ }^{1}$ han estado presentes en la literatura sobre la internacionalización de la empresa desde hace varias décadas; sin embargo, en los últimos años la investigación se ha centrado en ellas, permitiendo observar que una misma multinacional puede acoger filiales de distintos tipos (White y Poynter, 1984; Bartlett y Ghoshal, 1986, 1989; Jarillo y Martínez, 1990). Algunos investigadores (Andersson et al., 2001, 2002; Birkinshaw y Hood, 1997, 1998a), han presentado visiones dinámicas de las filiales, que no se limitan a la clasificación del rol que exhiben, sino que muestran la evolución de su estrategia, además de presentar los mecanismos que determinan esa evolución en el tiempo.

La influencia del entorno ha sido uno de los elementos tomados en cuenta al analizar la evolución de las filiales pero, aun cuando algunos estudios (Birkinshaw y Hood, 2000; Frost et al., 2002 y Birkinshaw et al., 1998) reconocen la importancia de darle una mayor consideración, el estudio de las diferentes condiciones del entorno ha sido escasamente abordado, puesto que los trabajos sobre roles de filiales se han desarrollado con base en el supuesto de que las filiales se encuentran en entornos locales estables $y$, en consecuencia, favorables al desarrollo de las actividades y operaciones de las mismas (White y Poynter, 1984;

1 En este trabajo se entiende por filial exterior de una Empresa Multinacional a aquella empresa localizada en un país distinto al de origen de la Multinacional cuya dirección y/o el control económico, financiero y administrativo depende en forma más o menos directa de la empresa que se denomina matriz, y que es propietaria del $50 \%$ o más de sus acciones. 
Bartlett y Ghoshal, 1986; Gupta y Govindarajan, 1991a, 1991b, 1994; Roth y Morrison, 1992; Birkinshaw y Morrison, 1995, entre otros).

De la revisión de la literatura que se realizó, sólo unos pocos trabajos, como los de Jarillo y Martínez (1990) y Jacque y Lorange (1984) tomaron en cuenta los efectos que puede causar un entorno desfavorable sobre las actividades de las filiales: los primeros presentan una tipología sobre filiales considerando las condiciones históricas particulares del país donde se realizó el estudio, España, y observan los efectos de haberse eliminado las barreras políticas que mantenían la economía española cerrada a la competencia internacional, mientras que los segundos hacen un estudio sobre filiales establecidas en economías hiperinflacionarias. De resto, los escasos aportes teóricos que se encuentran en la literatura especializada no es aplicable, puesto que no se consideran los diferentes niveles del entorno (Benito et al., 2003) o las diferencias en el desarrollo económico y social que existen entre los países desarrollados y las economías emergentes y en transición o las naciones más pobres (London y Hart, 2004; Hoskisson et al., 2000; Birkinshaw y Hood, 2000).

En este orden de ideas, las condiciones que enfrentan las filiales establecidas en países con entornos volátiles como Venezuela, país latinoamericano en vías de desarrollo, que por su evolución histórica y situación actual presenta condiciones económicas, sociales y políticas desfavorables para las actividades de las filiales exteriores, caracterizadas por importantes fluctuaciones de la economía, dependiente de una materia pri- ma básica como es el petróleo, una elevada intervención del Estado en la economía, debilidad del marco legal, entre otros aspectos que se evidencian claramente en indicadores de uso internacional como el de riesgo país (Santos y Villasmil, 2006; Garay, 2003; Sabal, 2002a).

Así, el objetivo de este trabajo es examinar la aplicación de los patrones de evolución de filiales propuestos por Birkinshaw y Hood (1998a) en entornos volátiles e indagar si las condiciones del entorno bajo las que se hace el estudio permiten establecer el patrón de evolución que impulsa el mismo entorno como factor determinante de la evolución del rol de las filiales dentro de las empresas multinacionales. Para ello, se presentan los tres grupos de factores que actúan como impulsores de la evolución de la filial: la asignación de la matriz, la iniciativa de la propia filial y, especialmente, el determinismo del entorno local. Los procesos de evolución de la filial se basan en la interacción entre la iniciativa estratégica y las capacidades de ésta. Utilizando la perspectiva de recursos (Wernerfelt, 1984; Barney, 1991; Amit y Schoemaker, 1993), se pretende analizar el tipo de iniciativas emprendidas por las filiales exteriores en entornos volátiles, así como las capacidades generadas en sus procesos de desarrollo.

\section{La evolución de la filial exterior y el papel del entorno}

Como el objetivo de este trabajo es avanzar en el análisis de los patrones de evolución de las filiales, con base en el planteamiento de que las filiales exteriores se pueden encontrar en un entorno 
volátil, a continuación se abordará teóricamente la idea sobre los que son entornos volátiles y su relación con las filiales, para luego revisar la literatura sobre los factores que impulsan la evolución de la filial y los procesos genéricos de evolución de las filiales propuestos por Birkinshaw y Hood (1998a), que servirán de referencia para presentar las cuestiones de investigación al final de esta sección del artículo.

\subsection{Entornos volátiles y las filiales}

Se debe tener en cuenta que cada filial exterior de una empresa multinacional se encuentra localizada en un país determinado y, que cada país ofrece a la filial un entorno con condiciones muy particulares y diferentes unas de otras. Esto implica, igualmente, que las filiales de una empresa multinacional estarán sometidas, al menos, a tantos entornos locales como países donde la multinacional tenga presencia.

Dentro de esta variedad, se pueden presentar casos de países que muestran unas condiciones favorables para el desarrollo y la realización de negocios, y otros, en los cuales esas mismas variables pueden ser negativas y resultar adversas para la realización actividades empresariales, la gestión de filiales exteriores y la realización de otras inversiones, como puede ocurrir cuando se examinan los Ilamados "mercados emergentes" de América Latina, Asia y Europa Oriental (Hoskisson et al., 2000; Penfold, 2002; Sabal, 2002b; Durán, 1999, 2001).

Esas condiciones a las que se hace referencia, son fundamentalmente de ca- rácter económico, tales como: la estabilidad de la economía, medida en términos de niveles de inflación, devaluación o sobrevaluación de la moneda, políticas fiscales y comerciales acertadas, el grado de desarrollo económico y social, aunque también pueden existir variables de carácter sociopolítico de peso como: el respeto por estado de derecho, que ofrezca seguridad jurídica a través de un marco legal claro y un sistema de administración de justicia eficaz, la estabilidad política y la gobernabilidad y, la fortaleza de sus instituciones (Durán, 2001; Hoskisson et al., 2000; Penfold, 2002).

Con base en las consideraciones de Johanson y Johanson (2006) sobre los entornos de las economías en transición y de Luo (2004) respecto a la elevada incertidumbre de las economías emergentes, se puede indicar que un entorno local volátil, es aquel que presenta condiciones políticas, económicas y sociales desfavorables para la operación de filiales exteriores de empresas multinacionales, caracterizados por indicadores negativos de variables económicas y sociopolíticas tales como: recesión o inestabilidad de la economía del país; elevada inflación; devaluación o sobrevaluación extrema de la moneda o el impedimento a la libre convertibilidad de ella; la volatilidad política o problemas de gobernabilidad; la corrupción y la inseguridad jurídica, entre otros, todos aspectos que pueden ser observados en diversos de indicadores de utilización internacional; por ejemplo, el riesgo país o el índice de percepción de la corrupción se mostrarían elevados, y el índice de libertad económica se tendería a ser más bien bajo. 


\subsection{Factores determinantes del rol de la filial}

La posición que ocupa una filial dentro de una multinacional es definido como "rol" de la filial por Galunic y Eisenhardt (1996), quienes lo definen como "el negocio, o elementos del negocio, en los que participa activamente y por los cuales tiene responsabilidad dentro de la corporación". Así, el tipo de rol desempeñado por la filial exterior es el resultado de la concurrencia de tres fuerzas que convergen en él: la asignación de la matriz, la iniciativa de la filial y el determinismo del entorno. La "asignación de la matriz" se refiere a la fijación de responsabilidades a la filial por parte de la matriz. La "iniciativa de la filial" implica que es la propia filial la que impulsa la adopción de un determinado rol, y recoge las decisiones tomadas por la dirección de la filial dentro del ámbito de sus competencias, para definir por ella misma el rol que asume. Finalmente, el tercer factor, denominado "determinismo del entorno local", implica que el rol de la filial puede ser alterado en función de las oportunidades y limitaciones que presenta el mercado local (Birkinshaw y Hood, 1998a).

Como se observa en la Figura 1 , los tres mecanismos interactúan de forma dinámica para determinar el rol de la filial. De este modo, éste condiciona y está condicionado por las decisiones adoptadas por los directivos de la matriz, las decisiones de los directivos de la propia filial y las condiciones del entorno local, que

\section{Figura 1}

Marco de organización de la determinación del rol de la filial

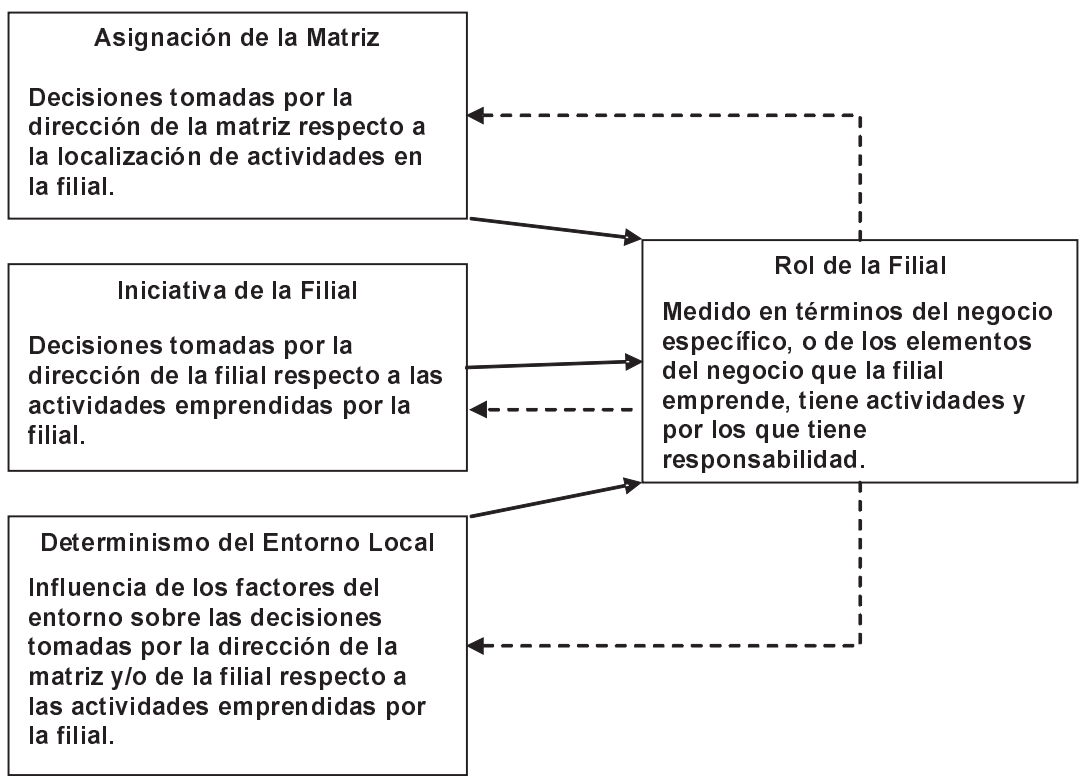

Fuente: Adaptado de Birkinshaw y Hood (1998a: 775). 
conducen a redefinir ese mismo rol de la filial en un momento dado (Birkinshaw y Hood, 1997, 1998a, 1998b; Hood y Taggart, 1999).

\subsection{Los procesos genéricos de evolución de las filiales}

A partir de la idea de que una filial puede a lo largo de su historia cambiar su rol y el nivel de capacidades con que cuenta en un momento dado, Birkinshaw y Hood (1998a) establecen cinco procesos genéricos de evolución de las filiales, que se explicarán a continuación. Sin embargo, esos procesos genéricos descritos por Birkinshaw y Hood (1998a), sólo consideran dos factores determinantes de la evolución de la filial: la asignación desde la filial y la iniciativa de la propia fi- lial, mientras que no se contempla al entorno como mecanismo que impulsa un cambio en el rol de la filial y, por lo tanto, de su evolución. La Figura 2 sintetiza los diferentes patrones de evolución del rol de las filiales que proponen Birkinshaw y Hood (1998a).

En el proceso genérico 1, la extensión del rol conduce, subsecuentemente, a una mejora del perfil de capacidades de la filial. En virtud de que la asignación del rol es responsabilidad de la matriz y que la filial todavía no ha generado las capacidades asociadas a aquel, se denomina a este proceso inversión impulsada por la matriz (IIM). Aunque los directivos de las filiales locales pudieran tener alguna influencia sobre el proceso (especialmente si muestran alto desempeño), típicamente estarán compitiendo activamen-

Figura 2

\section{Evolución de la filial según el cambio en sus capacidades y el rol} Cambio en el rol de la filial

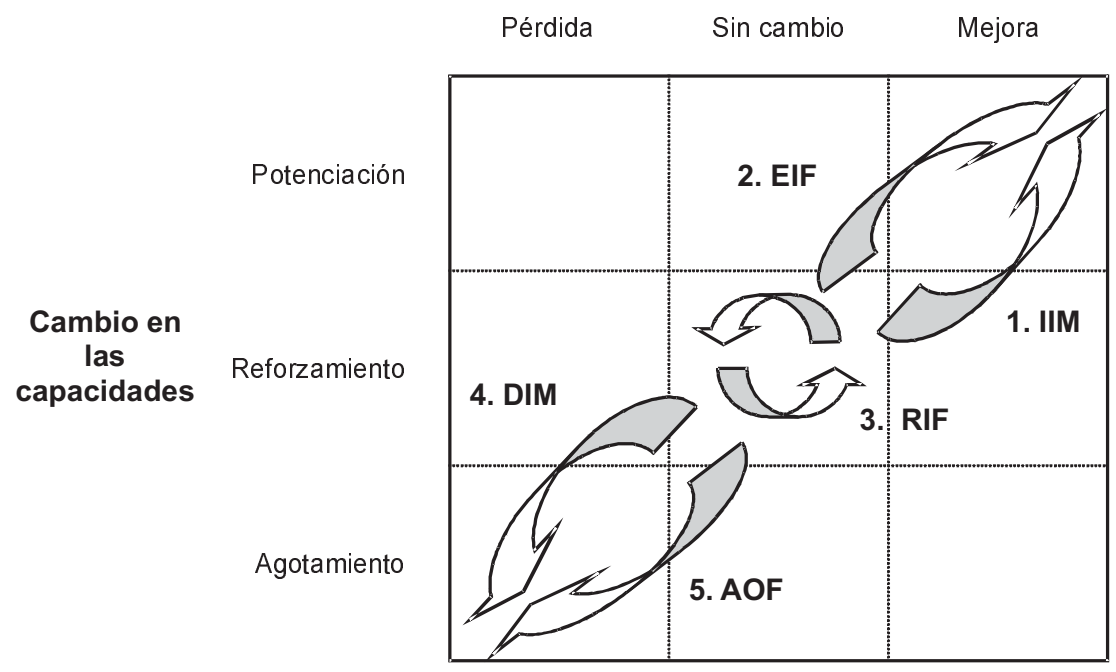

Fuente: Birkinshaw y Hood (1998a: 783). 
te contra otras filiales por hacerse con el rol, de manera que el desarrollo de las capacidades adecuadas comienza una vez que el rol ha sido asignado.

En la situación 2, el incremento de capacidades lidera una subsiguiente extensión del rol de la filial. Representa un movimiento estratégico de los directivos de la filial, que ven la posibilidad de mejorar su rol u obtener uno nuevo si pueden demostrar que tienen las capacidades necesarias para ello. Este proceso se denomina extensión del rol impulsada por la filial (EIF). Sin embargo, la modificación del rol no está garantizada en este caso, porque las capacidades desarrolladas pueden no ser valoradas por la dirección corporativa.

Los procesos 4 y 5 son el inverso de las situaciones 1 y 2 . La primera es la desinversión impulsada por la matriz (DIM), donde la filial pierde su rol respecto a cierto producto, tecnología o mercado, de forma que gradualmente las capacidades correspondientes se atrofian. EI otro caso es la atrofia por omisión de la filial (AOF), en el que las capacidades de la filial se van deteriorando gradualmente en el tiempo, su desempeño del rol sufre y la sede corporativa termina por retirarle el rol.

Por último, la situación 3 es el reforzamiento del rol impulsado por la filial (RIF), que se refiere al proceso en la que la filial agudiza o fortalece sus capacidades y mantiene su rol, en otras palabras, se consolida en su rol. Se puede pensar que éste no es propiamente un caso de evolución de filiales, pero los autores lo toman en cuenta por la situación en la que la filial opta por profundizar sus capacidades en un área específica en su actual rol, en lugar de buscar otros roles. Como parte de una estrategia a largo plazo, es importante realizar el reforzamiento del rol, porque asegura que la filial cuente con capacidades distintivas frente a competidores internos y externos. En este caso es más difícil, aunque no imposible, identificar cuando ha ocurrido la evolución, ya que el rol se mantiene en lugar de cambiar.

Así pues, en este trabajo se plantean las siguientes interrogantes de investigación:

1. ¿Será posible identificar todos los tipos de procesos de evolución de las filiales descritos por Birkinshaw y Hood (1998a) en entornos volátiles?

2. ¿Será posible distinguir algún proceso de evolución en las filiales que sea impulsado por el entorno?

\section{Metodología}

En la selección de la metodología de investigación a utilizar en el estudio, se han tomado en consideración las condiciones que lo caracterizaban y al comprobar que se reunian las condiciones que describen Eisenhardt (1989), Yin (1994) y Stake (1995) se ha llevado a cabo una metodología cualitativa basada en el estudio de casos. Esas condiciones son: permite entender mejor y con mayor profundidad cómo condicionan los entornos adversos a la determinación del rol de las filiales; no se tiene control sobre el fenómeno que se investiga, y los límites entre ese fenómeno y el medio en el que se desarrolla no están bien diferenciados.

Igualmente, se han tomado en consideración las condicionantes planteadas por Hoskisson et al. (2000), que respal- 
dan la utilización de la metodología cualitativa en este estudio. El primer aspecto que condiciona el estudio es que el estado de conocimiento sobre la cuestión a investigar es de desarrollo relativamente reciente y escaso (Bonache, 1999). El conocimiento en materia de roles estratégicos de las filiales exteriores de empresas multinacionales y sus factores determinantes es un área de desarrollo teórico reciente en el ámbito de la dirección de empresas internacionales. Son escasos los trabajos que han tomado en consideración el determinismo del entorno en el establecimiento de los roles de las filiales exteriores, y en la literatura no se han encontrado investigaciones que estudien el impacto del entorno en la determinación y evolución de los roles de esas filiales cuando éstas operan en países que presentan un entorno con características volátiles. En este sentido, la metodología cualitativa, en mejor medida que las herramientas estadísticas tradicionales, son particularmente útiles para explorar supuestos implícitos y examinar nuevas relaciones, conceptos abstractos y definiciones operativas (Stake, 1995; Bettis, 1991; Weick, 1996).

El segundo aspecto que condiciona este estudio se deriva de las consecuencias que implica la realización de su trabajo de campo en una economía emergente como lo sería la de Venezuela. La metodología cualitativa es particularmente útil en las investigaciones orientadas al estudio de estrategias en economías emergentes, debido a que las teorías promulgadas para las economías desarrolladas pueden no ser apropiadas para los países emergentes, o porque se enfrentan problemas metodológicos respecto a la recolección de datos, muestreo y medición de variables, y porque las economías emergentes no son un grupo homogéneo entre sí (Hoskisson et al., 2000; London y Hart, 2004; Prahalad y Lieberthal, 1998).

En ausencia de estadísticas oficiales de instituciones venezolanas, el dato más fiable que se obtuvo con relación a la primera de esas cuestiones se encontró en el World Investment Report 2009 que cifra el número de filiales de empresas multinacionales situadas en Venezuela en 545 para el año 2004 (UNCTAD, 2009).

Se consideró que el uso de múltiples casos sería la mejor estrategia para abordar esta investigación. En este sentido, para definir la unidad de análisis se utilizaron los siguientes criterios: Debían tratarse de filiales exteriores de empresas multinacionales de cualquier origen establecidas en Venezuela, cuya propiedad perteneciera mayoritariamente (más de $50 \%$ ) a corporaciones extranjeras. Las filiales podían realizar actividades de producción o limitarse al mercadeo y ventas de productos elaborados en otras partes del mundo por la multinacional.

La selección de los casos siguió criterios no probabilísticos, pues las filiales tenían que reunir diferentes condiciones: 1) tener distintas dimensiones; 2 ) operar en diferentes sectores económicos y, especialmente, que estuviera representado alguno de los sectores más importantes de Venezuela como lo serían el petróleo, gas, hierro o aluminio; 3 ) tener asignados diferentes roles estratégicos; $y, 4$ ) las multinacionales a las que pertenezcan deben seguir diferentes enfoques estratégicos.

En la selección se contactó con un total de 95 filiales, de las cuales se obtuvo 
aceptación en 41 casos para realizar las entrevistas y recabar información, lo que equivale a un $43,2 \%$ de filiales entrevistadas. En cada una de las filiales se realizaron entre una y tres entrevistas. La información obtenida se completó con documentación interna, fuentes externas, y en el $80 \%$ de los casos se tuvo acceso al perfil estratégico de la filial.

Para categorizar las filiales se tomó en cuenta una serie de factores como: la nacionalidad de origen de la multinacional, el sector económico y tipo de actividades de la filial, que son factores que permiten deducir el tipo de enfoque estratégico de la corporación y luego establecer el tipo de la filial, que sirvió para agruparlas. Se utilizó la tipología de Jarillo y Martínez (1990), que consideran dos dimensiones: el grado de integración de las actividades desarrolladas por una filial de un país determinado con las mismas actividades realizadas por otras filiales de la multinacional; y la localización geográfica de esas actividades, siendo que la localización en el país destino de actividades de valor, es una forma de sensibilizarse a las necesidades locales. Estos autores sólo identifican tres tipos de filiales: filiales Activas, Autónomas y Receptivas.

Las autónomas, se centran en el país donde están situadas, llevan a cabo la mayoría de las actividades de la cadena de valor, de tal forma que son relativamente independiente de la matriz y de otras filiales (Jarillo y Martínez, 1990). La filial es receptiva si sólo unas pocas actividades de la cadena de valor son realizadas en el país y están muy integradas con el resto de la empresa multinacional. Típicamente, se trata de las funciones de mercadeo y ventas. La filial es activa cuando, aunque realiza la mayor parte de las actividades de la cadena de valor en el país, son realizadas en estrecha coordinación con el resto de la multinacional, de tal forma que juega un papel destacado como nodo activo dentro de una red interorganizativa (Jarillo y Martínez, 1990).

\section{Discusión y aportes}

De las 41 filiales estudiadas en esta investigación, fueron identificadas 16 filiales autónomas, 13 filiales receptivas y 12 filiales activas, de acuerdo con la tipología de Jarillo y Martínez (1990). En el Cuadro 1, se describen algunos de los datos que permitieron identificar las tipologías de las filiales, y donde también se puede observar que los seudónimos que se utilizan para nombrarlas inician por letras que indican la tipología atribuida: A para las autónomas, $\mathrm{R}$ para las receptivas y $\mathrm{X}$ para las activas.

En el grupo de estudio, se observaron procesos de evolución y consolidación del rol de esas filiales, que fueron impulsados por la matriz y la filial, tal como fueron propuestos por Birkinshaw y Hood (1998a), pero también se encontraron evidencias que permitieron establecer que el entono es también un factor determinante de los procesos de evolución de las filiales con sentidos bien definidos. A continuación se examinarán los patrones encontrados para cada caso.

\subsection{Proceso de desarrollo del rol de la filial impulsado por la matriz}

Algunas filiales estudiadas resultaron favorecidas por las decisiones tomadas por la matriz en la ejecución de sus 


\section{Cuadro 1}

Datos de identificación de las tipologías de las filiales

\begin{tabular}{|c|c|c|c|c|}
\hline Filial & $\begin{array}{c}\text { Sector } \\
\text { de actividad }\end{array}$ & $\begin{array}{l}\text { Enfoque de la } \\
\text { Corporación }\end{array}$ & $\begin{array}{c}\text { Alcance } \\
\text { de Mercado }\end{array}$ & $\begin{array}{l}\text { Alcance } \\
\text { de Valor }\end{array}$ \\
\hline AAL & Alimentos & Multidoméstico & Limitado & Amplio \\
\hline ACA & Alimentos & Multidoméstico & Limitado & Amplio \\
\hline $\mathrm{ACO}$ & Construcción & Multidoméstico & Limitado & Amplio \\
\hline ACT & Contact Centers & Multidoméstico & Limitado & Amplio \\
\hline AEM & Empaques & Transnacional & Limitado & Amplio \\
\hline AEN & Eléctrico & Multidoméstico & Limitado & Amplio \\
\hline AGA & Agropecuario & Multidoméstico & Limitado & Amplio \\
\hline $\mathrm{AHA}$ & Alimentos & Multidoméstico & Limitado & Amplio \\
\hline AIN & Alimentos & Transnacional & Limitado & Amplio \\
\hline AIG & Consultoría & Transnacional & Amplio & Amplio \\
\hline ALA & Alimentos & Multidoméstico & Limitado & Amplio \\
\hline AMA & Consultoría & Transnacional & Amplio & Amplio \\
\hline AMO & Alimentos & Multidoméstico & Limitado & Amplio \\
\hline APA & Alimentos & Multidoméstico & Limitado & Amplio \\
\hline APR & Construcción & Multidoméstico & Limitado & Amplio \\
\hline ATE & Telecomunicaciones & Multidoméstico & Limitado & Amplio \\
\hline RAU & Automotriz & Transnacional & Limitado & Limitado \\
\hline RBE & Alimentos & Transnacional & Limitado & Limitado \\
\hline $\mathrm{RCO}$ & Consultoría & Global & Amplio & Limitado \\
\hline $\mathrm{RCM}$ & Alimentos & Transnacional & Limitado & Limitado \\
\hline RDU & Alimentos & Global & Limitado & Limitado \\
\hline RFA & Farmacéutico & Global & Limitado & Limitado \\
\hline RLA & Farmacéutico & Transnacional & Limitado & Limitado \\
\hline $\mathrm{RLI}$ & Alimentos & Global & Limitado & Limitado \\
\hline RME & Equipos médicos & Transnacional & Limitado & Limitado \\
\hline RPE & Petróleo & Global & Limitado & Limitado \\
\hline $\mathrm{RTI}$ & Tecnología & Global & Amplio & Limitado \\
\hline RTR & Automotriz & Global & Limitado & Limitado \\
\hline RVE & Equipos veterinarios & Global & Limitado & Limitado \\
\hline XAL & Aluminio & Transnacional & Amplio & Amplio \\
\hline $\mathrm{XCA}$ & Manufacturas & Transnacional & Amplio & Amplio \\
\hline $\mathrm{xCO}$ & Cosméticos & Transnacional & Amplio & Amplio \\
\hline
\end{tabular}




\section{Cuadro 1 (Continuación)}

\begin{tabular}{ccccc}
\hline Filial & $\begin{array}{c}\text { Sector } \\
\text { de actividad }\end{array}$ & $\begin{array}{c}\text { Enfoque de la } \\
\text { Corporación }\end{array}$ & $\begin{array}{c}\text { Alcance } \\
\text { de Mercado }\end{array}$ & $\begin{array}{c}\text { Alcance } \\
\text { de Valor }\end{array}$ \\
\hline XEL & Eléctrico & Transnacional & Amplio & Amplio \\
XFI & Serv. Financieros & Transnacional & Amplio & Amplio \\
XGT & Alimentos & Transnacional & Amplio & Amplio \\
XIN & Tecnología & Transnacional & Limitado & Amplio \\
XMA & Manufacturas & Transnacional & Amplio & Amplio \\
XMT & Materiales & Transnacional & Amplio & Amplio \\
XQM & Químico & Transnacional & Amplio & Amplio \\
XSA & Sanitarios & Transnacional & Amplio & Amplio \\
XSI & Tecnología & Transnacional & Limitado & Amplio \\
\hline
\end{tabular}

Fuente: Elaboración propia.

estrategias, lo que trajo como resultado un incremento de sus operaciones en el país de acogida. Las decisiones de la matriz pudieron estar basadas en una estrategia de racionalización global de la corporación (RTI y APR), en la adquisición de otras multinacionales a nivel mundial (RPE y XGT), o en la necesidad de hacer nuevas inversiones para incrementar la producción total de la multinacional (XSA) y/o la presencia de la filial en el país de acogida (ATE).

En los casos de RTI y APR, el resultado fue un incremento de su alcance de mercado como consecuencia del cierre de otras filiales de la región; las filiales RPE y XGT aumentaron sus operaciones locales al añadírseles las actividades de las filiales de la multinacional adquirida y XSA recibió inversiones para duplicar su capacidad de producción a fin de concretar la estrategia de la corporación de aumentar sus exportaciones a los Estados Unidos de América. Finalmente, ATE ha recibido constante respaldo económico para incrementar su cobertura y oferta de servicios en el mercado local.

\subsection{Proceso de desarrollo del rol impulsado por la filial}

Las filiales estudiadas emprendieron varios tipos de iniciativas a fin de desarrollar su rol, entre las cuales, además de algunas de las citadas por Birkinshaw (1995) y Delany (1998; 2000), se debe agregar la reducción de la línea de reporte.

- Búsqueda de nuevas oportunidades de negocio en el mercado local: las filiales XFI, XIN, XSI, AAL y ALA desarrollaron iniciativas para incrementar su portafolio de negocios en el país de destino. XFI ha desarrollado toda una gama de servicios adicionales al negocio inicial, que actualmente sólo representa un $10 \%$ de sus ingresos por ventas. XIN ha desarrollado servicios complementarios a su actividad principal (procesamiento documental de cheques). XSI desarrolló 
capacidades en una nueva área de negocios para la multinacional (call centres) y se encuentra difundiéndola a otras filiales de la corporación. AAL se involucró en el negocio de los cereales para bebé y alimentos para mascotas; y, ALA hizo lo propio con los zumos de frutas y quesos.

- Extensión del mandato: las filiales XSA, XAL y XCO mejoraron su rol, al incorporar o incrementar las actividades de investigación y desarrollo en sus operaciones. De igual forma, a RLI se le asignó la administración de las operaciones de la corporación en Colombia y AMO consiguió que se instalara una nueva planta de producción para el procesamiento local de un mayor número de productos. En el área de marketing, XCA y XGT desarrollaron productos que se comercializan internacionalmente, y XMT se ha esforzado en producir, comercializar y promover en tiempo record nuevos productos para compensar los efectos de las regulaciones de precios en el país de acogida. En otros casos, las filiales han introducido de forma rápida y eficaz nuevas líneas de productos de la corporación (AMO).

Para algunas de las filiales estudiadas, las posibilidad de desarrollar su rol se encontraba abriendo espacios en el área internacional. En este sentido, ACA fue promotora de la creación de nuevas filiales en otros países de la región: Colombia y Ecuador y se convirtió en la sede regional de la corporación, reportando los resultados consolidados de las tres filiales a la matriz. XFI estableció una nueva filial en Re- pública Dominicana y está próxima de abrir otra en Centroamérica. AMA y AIG se internacionalizaron exportando sus servicios y, de igual manera, XCA también extendido su rol con una mayor internacionalización de su producción.

- Reconfiguración de las operaciones existentes: XCO consiguió beneficiarse en un proceso en el que la matriz quería reubicar la unidad de coordinación de exportaciones de Latinoamérica; con ese proceso de racionalización de las operaciones internacionales de la multinacional, las responsabilidades de coordinación de las exportaciones de Latinoamérica, que antes estaban en Puerto Rico pasaron a Venezuela. De igual manera, RCO expandió su alcance de mercado para ocupar otras áreas geográficas que antes eran atendidas por otras filiales. XEL también ha obtenido un mayor alcance de mercado al conseguir responsabilidades sobre una región que antes era gestionada por la corporación.

- Reducción de la línea de reporte: algunas filiales han logrado ser nombradas sede regional de la multinacional (ACA, APR, RTI, RCO, RBE, XCO y $X M T$ ), con lo cual, han mejorado su posición en la línea de reporte ante la matriz e incrementado su poder de influencia sobre las decisiones de la corporación.

\subsection{Proceso de desarrollo del rol impulsado por el entorno}

En esta investigación, los procesos de desarrollo que han sido impulsados por el entorno han reforzado o extendido 
el rol de la filial. Este proceso se podría denominar Mejora del rol impulsado por el entorno (MIE). En este caso, el entorno local ejerce su influencia sobre las decisiones tomadas por la dirección de la matriz y/o de la filial respecto a las actividades emprendidas por la filial (Birkinshaw y Hood, 1998a). Visto así, es un impulsor que ejerce su influencia de forma indirecta sobre el rol de la filial. Pero en algunos casos, las filiales pueden recibir un impulso en su rol gracias a la actuación directa de la administración local, quien mediante políticas públicas puede cambiar la situación de sectores y empresas en particular.

Por ejemplo, una de las formas que dispone la administración del país de acogida para impulsar el rol de la filial exterior es a través de las compras gubernamentales, como ocurre en los casos de ACO, RME y RTR. En otros casos, la filial resulta beneficiada por la aplicación de políticas económicas por parte del gobierno, bien porque el sector en el que participa la filial recibe consideraciones especiales por parte del gobierno, como es el caso del sector petrolero en Venezuela, que beneficia a la filial RPE y el sector eléctrico, que beneficia a $\mathrm{ACO}$, o bien porque las políticas cambiarias, arancelarias o comerciales fijadas por el gobierno le es favorable a la filial, como serían los casos de las filiales RME, RTR y RPE. Es importante indicar que el beneficio en el rol de las filiales depende del mantenimiento de la actuación de la administración local, y que un cambio de sentido en las decisiones políticas puede causar un cese en el desarrollo del rol de la filial o, en el peor de los casos, puede ocasionar un cambio hacia el declive del rol.

\subsection{Proceso de consolidación de las filiales}

Como se señaló en el apartado 2.3, el proceso de consolidación del rol depende enteramente de las iniciativas de la filial local, que responde ante la competencia de filiales hermanas y competidores externos (Birkinshaw y Hood, 1998a; Crookell y Morrison, 1990).

Las filiales estudiadas en esta investigación, se concentraron en iniciativas que les permitieran consolidar su actual rol. Con ese fin, además de iniciativas orientadas al mejoramiento del desempeño y la participación en decisiones de la matriz, también emprendieron acciones que les permitieran ganar tamaño mientras ejecutan su actual rol, para mejorar su importancia relativa dentro de sus corporaciones, que luego pueda permitirles una evolución hacia el desarrollo.

- Mejora del desempeño: las filiales se han dado a la tarea de mejorar su competitividad en costes, calidad y, especialmente, rentabilidad para mantenerse en su rol. Destacan en rentabilidad las filiales: ACT, ALA, AAL, AHA, AMO, APA, XCA, XGT, RBE, RLI, RCM y RDU. Otras filiales presentan excelentes indicadores generales de gestión: XIN, XSI, XMT, XMA, XAL, $X C O, A E M, R C O, R L A$ y RTI.

- Participación en las decisiones corporativas: algunas de las filiales estudiadas incrementaron su participación en decisiones corporativas. En algunos casos ello ha implicado la "exportación" de recursos humanos de la filial a la matriz o a otras unidades de la corporación (XMA, XMT, 
ACA, AAL, XFI, RTI, y RLI), la participación en proyectos de alcance global (ACA, RBE y AIN), la transferencia de tecnología y mejores prácticas (áreas de marketing, ventas, distribución, sistemas de información y operaciones) a otras unidades de la corporación (XMT, XMA, XIN, AMA, AHA, ATE, ACT, RLI, RCM, RDU y RBE) y el lograr que negocios en los que participa actualmente la filial sean considerados por la matriz como estratégicos para toda la corporación (ALA lo hizo con el negocio de los zumos de frutas y AHA con el negocio de las pastas alimenticias).

- Aumento de tamaño: aunque siguen realizando las mismas actividades sin cambiar su alcance de producto y/o mercado, algunas filiales se han dedicado a incrementar sus operaciones en el país de acogida, realizando nuevas inversiones en adquisición de empresas locales del mismo sector de la filial (ALA) y en la ampliación de sus capacidades de producción (ALA, AHA, AMO, XSA y XCA).

\subsection{Procesos de declive de las filiales}

Al igual que en los procesos de desarrollo, en los procesos de declive del rol de las filiales se evidencia la participación de todos los determinantes del rol de las filiales: la matriz, la filial y el entorno.

Declive del rol impulsado por la matriz

Los procesos de declive de los roles de las filiales que son impulsados por la matriz están relacionadas usualmente con estrategias y políticas corporativas que se aplican en toda la multinacional y que, a veces, implican cambios radicales en el rol de una filial. Como consecuencia, algunas de las filiales que resultan perjudicadas sufren un declive muy fuerte en sus roles: en los casos particulares de RLI y RBE, las filiales debieron dejar sus actividades de producción y sus roles pasaron de ser activas o autónomas a receptivas de marketing y ventas, con lo cual, las actividades y funciones desarrolladas por estas filiales se empobrecieron cuantitativa y cualitativamente.

En el grupo de filiales estudiadas, el declive de una filial fue impulsado por la matriz debido a las diferentes razones que se exponen a continuación:

\section{- Reorganización de la producción:}

RLI dejó sus actividades de producción para ser asumidas por otra división de la multinacional, como parte de un proceso de reorganización en el que la corporación separó las actividades de producción de las de marketing y ventas en el país de acogida. A RLI se le dejó las actividades de marketing y ventas y otra filial racionalizada, dependiente de una división de aprovisionamiento mundial, se encarga de las actividades de producción en el país de acogida.

- Cambios de estrategia de la corporación: Ante un cambio de estrategia de la matriz, que decidió focalizar sus esfuerzos en menos productos de marcas fuertes a nivel internacional, RBE debió desprenderse de sus activos de producción porque estaban destinados a la fabricación de un pro- 
ducto con una marca local que no se encontraba en línea con la estrategia corporativa. La matriz de AEN ha rechazado oportunidades de negocios porque no quiere nuevos negocios en Venezuela, a pesar de que la filial tiene un excedente en la generación de electricidad que se está perdiendo. ALA debió suspender sus actividades de investigación y desarrollo a favor de la matriz, porque un cambio de estrategia reorientó esta función hacia la concentración en la sede corporativa. RDU perdió su rol como líder de su región como consecuencia de una reorganización por áreas geográficas que realizó la matriz.

Declive del rol impulsado por la filial

El declive del rol impulsado por la filial se refiere a una pérdida de rol debido a que la filial posee un nivel relativo de capacidades menor que alguna de las filiales hermanas y, en un proceso de racionalización global, pierde el rol aquella que tenga menos capacidades (Birkinshaw y Hood, 1998a).

Esta alternativa para el declive de la filial se puede observar en uno de los casos del grupo de filiales estudiado (RCM) y se produce aunque la filial mantuvo adecuadamente sus capacidades para cumplir con su rol pero, ante un programa de racionalización global aplicado por la multinacional, resultó que la filial RCM tenía, relativamente, menos capacidad de producción que su hermana de Colombia. El resultado fue que las operaciones de producción de Venezuela fueron suspendidas y en Colombia fueron incrementadas.
Declive del rol impulsado por el entorno

En esta investigación se han podido registrar procesos de declive del rol de las filiales que han estado impulsados por el entorno. Este proceso de evolución podría ser llamado Declive del rol impulsado por el entorno (DIE). Al igual que en el Desarrollo del rol impulsado por el entorno, se observa la influencia de los factores del entorno sobre las decisiones tomadas por la dirección de la matriz y/o de la filial respecto a las actividades emprendidas por la filial, a veces evidenciada de forma directa o otras indirectamente. Por ejemplo, en unos casos el declive ha sido causado por la disminución de la demanda, producto de la inestabilidad de la economía, pero en otros casos, el declive se ha debido a modificaciones en el marco legal y un cumplimiento deficiente del ordenamiento jurídico en el país de acogida, o problemas sectoriales originados por la intervención del Estado o la aplicación políticas públicas incoherentes e inconsistentes en el tiempo, que no han servido para ofrecer los incentivos que requiere el sector para desempeñarse adecuadamente.

En este sentido, las filiales XEL, RVE y RAU sufrieron un descenso muy importante en sus operaciones debido a la disminución de la demanda; en los casos de XEL y RVE, la matriz consideró cerrar la filial y, la situación de RAU pasó por una etapa crítica que duró más de un año durante el cual cerró la planta de ensamblaje y se dedicó únicamente a la prestación de servicio postventa. La filial XSI pasó igualmente por una situación difícil debido a una contracción de su principal mercado, y un caso similar ocurrió con la filial AIG. 
Los roles de las filiales AGA y AEN han declinado como consecuencia del cumplimiento deficiente de las regulaciones legales. En el caso de AGA, problemas de seguridad jurídica le han impedido mantener sus operaciones a un nivel normal debido a la intervención de sus activos de producción, mientras que en el caso de AEN y XEL, la falta de aplicación (ejecución por parte de la administración local) de la ley que rige su sector es lo que ha representado el mayor problema.

Los problemas sectoriales afectaron a las empresas cuyos mercados están vinculados con esos sectores: a AEN y XEL las afectó los problemas del sector eléctrico, y a RVE y AGA, los del sector agrícola.

Así, mientras las condiciones del entorno se mantengan inalteradas, el rol de la filial mantendrá el declive. Pero una rectificación en las políticas públicas y un cambio en la actuación de la administración local pueden detener el declive del rol de la filial y, en el mejor de los casos, puede impulsar el desarrollo del rol.

La Figura 3 presenta la evolución de las filiales según los cambios en sus capacidades y el rol incorporando los patrones de evolución que responden al impulso del entorno según fueron identificados, y a los que se denominó Mejora del rol impulsado por el entorno (MIE) y Declive del rol impulsado por el entorno (DIE).

\subsection{Evolución dinámica de los roles}

En el Cuadro 2, se puede observar un resumen de los diferentes procesos de evolución de las filiales estudiadas, de

\section{Figura 3}

\section{Extensión de los patrones de evolución de la filial de Birkinshaw y Hood}

\section{Cambio en el rol de la filial}

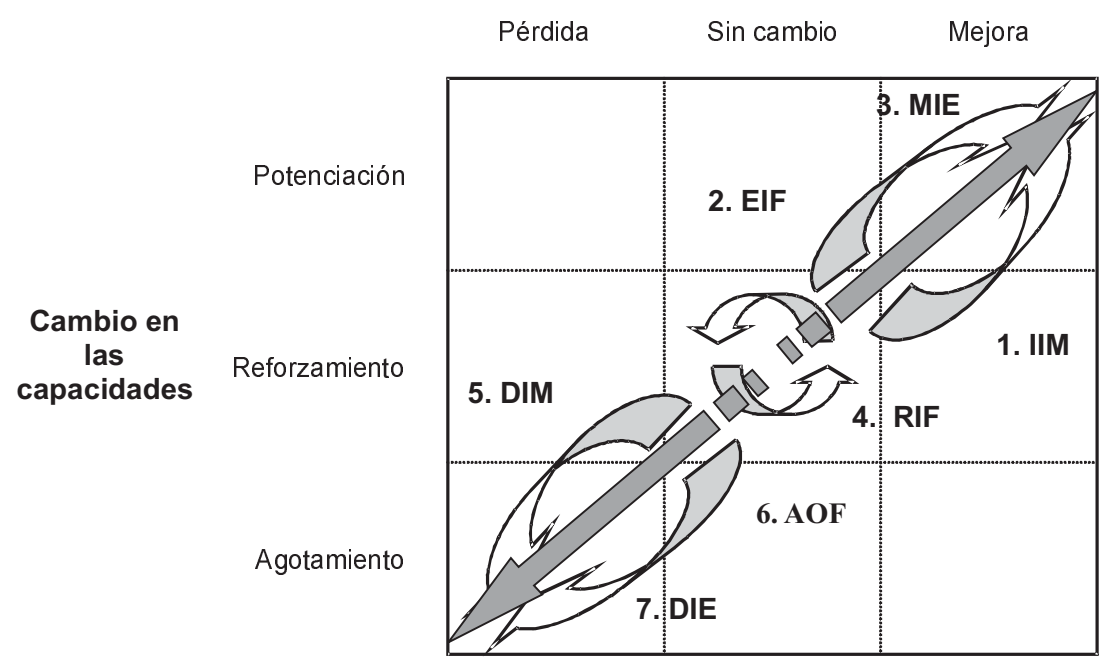

Fuente: Adaptado de Birkinshaw y Hood (1998a). 


\section{Cuadro 2 \\ Evolución de las filiales en entornos volátiles}

\begin{tabular}{|c|c|c|}
\hline Evolución & Tipo de impulsor & Filiales \\
\hline \multirow{3}{*}{$\begin{array}{l}\text { Desarrollo del rol } \\
\text { impulsado por la matriz }\end{array}$} & Nuevas inversiones. & XSA y ATE. \\
\hline & $\begin{array}{l}\text { Adquisición de otra } \\
\text { multinacional. }\end{array}$ & RPE y XGT. \\
\hline & Estrategias de racionalización. & RTI y APR. \\
\hline \multirow{4}{*}{$\begin{array}{l}\text { Desarrollo del rol } \\
\text { impulsado por la filial }\end{array}$} & $\begin{array}{l}\text { Búsqueda de nuevas } \\
\text { oportunidades de negocio en el } \\
\text { mercado local. }\end{array}$ & XFI, XIN, XSI, AAL y ALA. \\
\hline & Extensión del mandato. & $\begin{array}{l}\text { XSA, XAL, XCO, XMT, XCA, } \\
\text { XGT, XFI, RLI, AMO, ACA, AMA } \\
\text { y AIG. }\end{array}$ \\
\hline & $\begin{array}{l}\text { Reconfiguración de las } \\
\text { operaciones existentes. }\end{array}$ & XCO, XEL y RCO. \\
\hline & $\begin{array}{l}\text { Reducción de la línea de } \\
\text { reporte. }\end{array}$ & $\begin{array}{l}\text { ACA, APR, RTI, RCO, RBE, } \\
\text { XCO y XMT. }\end{array}$ \\
\hline \multirow{2}{*}{$\begin{array}{l}\text { Desarrollo del rol } \\
\text { impulsado por el entorno. }\end{array}$} & Adquisiciones gubernamentales. & ACO, RME y RTR. \\
\hline & $\begin{array}{l}\text { Políticas públicas en materia } \\
\text { económica. }\end{array}$ & RPE, RME, RTR y ACO. \\
\hline \multirow{3}{*}{$\begin{array}{l}\text { Consolidación del rol } \\
\text { impulsado por la filial }\end{array}$} & Mejora del desempeño. & $\begin{array}{l}\text { XIN, XSI, XMT, XMA, XAL, XCA, } \\
\text { XGT, XCO, ACT, ALA, AAL, } \\
\text { AHA, AMO, APA, AEM, RCO, } \\
\text { RLA, RTI, RBE, RLI, RCM y } \\
\text { RDU. }\end{array}$ \\
\hline & $\begin{array}{l}\text { Participación en las decisiones } \\
\text { corporativas. }\end{array}$ & $\begin{array}{l}\text { XMA, XMT, XIN, XFI, ACA, ALA, } \\
\text { AHA, AAL, AIN, AMA, ATE, } \\
\text { ACT, RLI, RCM, RTI, RDU y } \\
\text { RBE. }\end{array}$ \\
\hline & Aumento de tamaño. & ALA, AHA, AMO, XSA y XCA. \\
\hline \multirow{2}{*}{$\begin{array}{l}\text { Declive del rol impulsado } \\
\text { por la matriz }\end{array}$} & $\begin{array}{l}\text { Reorganización de la } \\
\text { producción. }\end{array}$ & RLI. \\
\hline & $\begin{array}{l}\text { Cambios de estrategia de la } \\
\text { corporación. }\end{array}$ & ALA, AEN, RBE y RDU. \\
\hline $\begin{array}{l}\text { Declive del rol impulsado } \\
\text { por la filial }\end{array}$ & $\begin{array}{l}\text { Racionalización de la } \\
\text { producción. }\end{array}$ & RCM. \\
\hline \multirow{3}{*}{$\begin{array}{l}\text { Declive del rol impulsado } \\
\text { por el entorno }\end{array}$} & Contracción de la demanda. & RVE, RAU, AIG, XSI y XEL. \\
\hline & Incumplimiento del marco legal. & AGA, AEN y XEL. \\
\hline & Problemas sectoriales. & AGA, RVE, XEL y AEN. \\
\hline
\end{tabular}

Fuente: Elaboración propia. 
acuerdo con el tipo de impulso que recibieron. Se encuentra que los cambios más numerosos fueron hacia la consolidación del rol, seguidos por procesos que condujeron hacia el desarrollo del rol y, finalmente, los que llevaron a la filial al declive. También se aprecia que la iniciativa de la filial fue el impulsor que más contribuyó a la evolución de los roles, concentrado básicamente en la consolidación, seguido por las iniciativas que buscan el desarrollo de la filial.

Luego, se observa que el entorno fue el segundo mayor impulsor de los procesos de evolución de las filiales estudiadas, donde se destaca que la mayor parte de cambios de rol fueron hacia el declive de la filial. Por último, le siguen los procesos de evolución impulsados por la matriz que, aunque fueron los menores en número, tuvieron el mayor impacto en lo que se refiere a los cambios radicales de rol porque implicaron saltos de tipologías en algunas de filiales involucradas, especialmente cuando se orientaron hacia procesos de declive.

En los procesos impulsados por la matriz se observó una ligera tendencia a favorecer más los procesos hacia el desarrollo de la filial, aunque los cambios más radicales de rol se registraron hacia el declive. Sin embargo, se debe comentar que las razones de la matriz para impulsar el cambio de rol de las filiales, hacia el desarrollo o bien hacia declive, se debió más a razones de estrategia global que a los efectos de la volatilidad del entorno local. Una explicación que permite comprender esta situación se encuentra si se piensa más en lo que no hace la matriz en lugar de observar lo que si hace, en el sentido de que, posiblemente, el papel de la matriz como instancia de aprobación de propuestas de la filial sea más importante que su papel como impulsora activa de cambios de rol. Así, podría entenderse que la volatilidad del entorno afectará más la aprobación de propuestas de las filiales, porque la matriz las rechazará en un mayor número de ocasiones (Birkinshaw y Ridderstrale, 1999; Birkinshaw y Fry, 1998). Estos hallazgos establecen que la filial tiende a impulsar un mayor número de procesos de evolución, pero con menor impacto de los que determina la matriz.

Además, en concordancia con las ideas de Birkinshaw (1996), muestran que una misma filial puede cambiar de rol varias veces y en diferentes sentidos, ganando y perdiendo roles. En cuatro de los casos que se estudiaron (RCM, RLI, RBE y XSI), las pérdidas de rol estaban seguidas por procesos de desarrollo. Los procesos de declive de las filiales RCM, RLI y RBE tuvieron una gran magnitud y conllevaron a cambios de tipología; en cambio, en el caso de la pérdida de rol de la filial XSI, el efecto fue menos importante. Por otro lado, se observa que el impulso hacia el desarrollo de las filiales fue diferente entre ellas, aunque el determinante en todas fue su propia iniciativa: en las filiales RCM, RLI y RBE fue más reducido, mientras que en el caso de XSI fue de una mayor proporción.

Estas diferencias pueden tener posibles explicaciones: primero, el declive es mayor cuando es impulsado por la matriz que cuando es inducido por el entorno; segundo, el nivel de capacidades de las filiales era diferente y la filial XSI tenía un mayor nivel de recursos y capacidades que las demás; $y$, finalmente, tam- 
bién puede existir una tercera explicación que combine las dos anteriores, en el sentido de que como el declive de las filiales fue mayor en los casos de RCM, RLI y RBE, ello les dejó con una menor dotación de capacidades que pudieran ser aplicadas luego al desarrollo del rol, en tanto que como en la filial XSI, el declive fue menor, sus capacidades básicas se mantuvieron y ello permitió que impulsara un desarrollo del rol con mayor alcance. Este último argumento estaría de acuerdo con la posición de Delany (2000), según la cual, la posibilidad de que una filial pueda desarrollar exitosamente una iniciativa dependerá del punto estratégico del que parte. Visto de otro modo, la posición estratégica de las filiales está relacionada con la dotación de capacidades con las que cuenta de manera que, una mejor posición estaría relacionada con una mayor base de capacidades.

\section{Conclusiones}

Los resultados de esta investigación confirman que el entorno es un impulsor de la evolución del rol de las filiales tan importante como el determinismo de la matriz y la iniciativa de la propia filial. Asimismo, se evidencia que el impacto del entorno puede ser tanto positivo como negativo cuando se trata del futuro de las filiales. Mas aún, contrario a lo que podía esperarse, es posible que en entornos volátiles como el de Venezuela, el efecto del entorno sobre algunas filiales puede ser positivo. Por otro lado, confirma la validez de los patrones genéricos de evolución de las filiales de Birkinshaw y Hood (1998a), y los extiende al incorporar dos nuevos patrones en la evolución de las fi- liales que son los impulsados por el entorno, con lo cual se completa el modelo organización de la determinación del rol de la filial de Birkinshaw y Hood (1998a), en el que los determinantes del rol de las filiales incorporan al entorno además de la asignación de la matriz y la iniciativa de la filial.

El elemento adversidad en el entorno se manifestó de forma diferenciada para las filiales, en parte debido a la discrecionalidad que conlleva la acción de las administraciones públicas y en parte por las características del sector en el que compite cada filial, pero fue clave para evidenciar patrones de evolución en distintos sentidos, bien sea hacia el desarrollo como hacia el declive. En países donde las condiciones del entorno son estables y favorables habría sido más difícil hacer este hallazgo.

La receptividad que muestre la matriz hacia las iniciativas emprendidas por la filial dependerá de su estrategia de competencia internacional, pero en cualquier caso, está claro que la iniciativa es un elemento clave para explicar la evolución de la filial. Aun cuando se trate de decisiones adoptadas por la matriz, la competencia y rivalidad entre filiales hermanas forma parte de los criterios subyacentes a la hora de asignar recursos a las filiales, por lo que las capacidades distintivas con que cuente cada filial se tomarán en consideración en la mayoría de decisiones que afecten a su desarrollo.

Aunque la propia iniciativa de las filiales fue el determinante para su desarrollo que se presentó con mayor frecuencia, se observó que el impulso en ese sentido tuvo diferente grado de magnitud entre ellas, probablemente asociados 
con la dotación de recursos y capacidades de cada una de ellas y siempre se encontró que las pérdidas de roles estaban seguidos por procesos de desarrollo. Desgranar las condiciones específicas en las que se gestan las iniciativas y categorizar con mayor nivel de desagregación el rango de capacidades que puede desarrollar una filial en entornos volátiles supone también un reto para futuras investigaciones.

\section{Referencias bibliográficas}

Amit, Raphael y Schoemaker, Paul J. H. (1993). Strategic assets and organizational rent. Strategic Management Journal, Vol. 14, No. 1, pp. 3346.

Andersson, Ulf, Forsgren, Mats y Holm, Ulf (2001). Subsidiary Embeddedness and Competence Development in MNCs-Multi-level Analysis. Organization Studies, Vol. 22, No. 6, pp. 1013-1034.

Andersson, Ulf, Forsgren, Mats y Holm, Ulf (2002). The strategic impact of external networks: subsidiary performance and competence development in the multinational corporation. Strategic Management Journal, Vol. 23, No. 11, pp. 979-996.

Barney, Jay B. (1991). Firm Resources and Sustained Competitive Advantage. Journal of Management, Vol. 17, No. 1, pp. 99-120.

Bartlett, Christopher A. y Ghoshal, Sumantra (1986). Tap your subsidiaries for global reach. Harvard Business Review, Vol. 64, No. 6, EUA, Harvard University, pp. 87-94.

Bartlett, Christopher A. y Ghoshal, Sumantra (1989). Managing across borders. The transnational solution. Harvard Business School Press, Boston.
Benito, Gabriel R. G., Grogaard, Birgitte y Narula, Rajneesh (2003). Environmental influences on MNE subsidiary roles: economic integration and the Nordic countries. Journal of International Business Studies, Vol. 34, No. 5, pp. 443-456.

Bettis, Richard A. (1991). Strategic management and the straightjacket: an editorial essay. Organization Science, Vol. 2, No. 3, pp. 315-319.

Birkinshaw, Julian (1995). Taking the initiative. Business Quarterly, Vol. 59, No. 4, pp. 97-102.

Birkinshaw, Julian (1996). How Multinational Subsidiary Mandates are Gained and Lost. Journal of International Business Studies, Vol. 27, No. 3, pp. 467-495.

Birkinshaw, Julian y Fry, Nick (1998). Subsidiary Initiatives to Develop New Markets. Sloan Management Review, Vol. 39, No. 3, pp. 51-61.

Birkinshaw, Julian y Hood, Neil (1997). An Empirical Study of Development Processes in Foreign-owned Subsidiaries in Canada and Scotland. Management International Review, Vol. 37, No. 4, pp. 339-364.

Birkinshaw, Julian y Hood, Neil (1998a). Multinational Subsidiary Evolution: Capability and Charter Change in ForeignOwned Subsidiary Companies. Academy of Management Review, Vol. 23, No. 4, pp. 773-795.

Birkinshaw, Julian y Hood, Neil (1998b). Multinational corporate evolution and subsidiary development. Macmillan Press, Londres.

Birkinshaw, Julian y Hood, Neil (2000). Characteristics of Foreign Subsidiaries in Industry Clusters. Journal of International Business Studies, Vol. 31, No. 1, pp. 141-154.

Birkinshaw, Julian, Hood, Neil y Jonsson, Stefan (1998). Building firm-specific ad- 
vantages in multinational corporations: the role of subsidiary initiative. Strategic Management Journal, Vol. 19, No. 3, pp. 221-241.

Birkinshaw, Julian y Morrison, Allen (1995). Configurations of strategy and structure in subsidiaries of multinational corporations. Journal of International Business Studies, Vol. 26, No. 4, pp. 729-753.

Birkinshaw, Julian y Ridderstrale, Jonas (1999). Fighting the corporate immune system: a process study of subsidiary initiatives in multinational corporations. International Business Review, Vol. 8, No. 2, pp. 149-180.

Bonache, Jaime (1999). El estudio de casos como estrategia de construcción teórica: características, críticas y defensas. Cuadernos de Economía y Dirección de la Empresa, No. 3, España, Asociación Científica de Economía y Dirección de la Empresa, ACEDE, pp. 123-140.

Crookell, Harold H. y Morrison, Allen (1990). Subsidiary Strategy in a Free Trade Environment. Business Quarterly, Vol. 55, No. 2, pp. 33-39.

Delany, Ed (1998). Strategic Development of Multinational Subsidiaries in Ireland. En Birkinshaw, Julian y Hood, Neil: Multinational corporate evolution and subsidiary development, pp. 239-267. Macmillan Press, Londres.

Delany, Ed (2000). Strategic Development of the Multinational Subsidiary through Subsidiary Initiative-taking. Long Range Planning, Vol. 33, No. 2, pp. 220-244.

Durán, Juan José (1999). Multinacionales Españolas en Iberoamérica: valor estratégico. Ediciones Pirámide, Madrid.

Durán, Juan José (2001). Estrategia y economía de la empresa multinacional. Ediciones Pirámide, Madrid.
Eisenhardt, Kathleen M. (1989). Building Theories from Case Study Research. Academy of Management Review, Vol. 14, No. 4, pp. 532-550.

Frost, Tony S., Birkinshaw, Julian M. y Ensign, Prescott C. (2002). Centers of Excellence in Multinational Corporations. Strategic Management Journal, Vol. 23, No. 11, pp. 997-1018.

Galunic, D. C. y Eisenhardt, Kathleen M. (1996). The Evolution of Intracorporate Domains Divisional Charter Losses in High-technology, Multidivisional Corporations. Organization Science, Vol. 7, No. 3, pp. 255-282.

Garay, Urbi (2003). La inestabilidad política y el riesgo país de Venezuela. En Márquez, Patricia y Piñango, Ramón: En esta Venezuela: realidades y nuevos caminos, pp. 113-124. Ediciones IESA, Caracas.

Gupta, Anil y Govindarajan, Vijay (1991a). Knowledge flows and the structure of control within multinational corporations. Academy of Management Review, Vol. 16, No. 4, pp. 768-792.

Gupta, Anil y Govindarajan, Vijay (1991b). Knowledge Flow Patterns, Subsidiary Strategic Roles, and Strategic Control within MNCs. Academy of Management Proceedings, pp. 21-25.

Gupta, Anil y Govindarajan, Vijay (1994). Organizing for Knowledge Flows within MNCs. International Business Review, Vol. 3, No. 4, pp. 443-457.

Hood, Neil y Taggart, James H. (1999). Subsidiary Development in German and Japanese Manufacturing Subsidiaries in the British Isles. Regional Studies, Vol. 33 , No. 6, pp. 513-528.

Hoskisson, Robert E., Eden, Lorraine, Lau, Chung Ming y Wright, Mike (2000). Strategy in Emerging Economies. Academy of Management Journal, Vol. 43, No. 3, pp. 249-267. 
Jacque, Laurent L. y Lorange, Peter (1984). The International Control Conundrum: The Case of 'Hyperinflationary' Subsidiaries, Journal of International Business Studies, Vol. 15, No. 2, pp. 185-201.

Jarillo, J. Carlos y Martínez, Jon I. (1990). Different roles for subsidiaries: the case of multinational corporations in Spain. Strategic Management Journal, Vol. 11, No. 7, pp. 501-512.

Johanson, Martin y Johanson, Jan (2006). Turbulence, Discovery and Foreign Market Entry: A Longitudinal Study of an Entry into the Russian Market. Management International Review, Vol. 46 , No. 2, pp. 179-205.

London, Ted y Hart, Stuart L. (2004). Reinventing strategies for emerging markets: beyond the transnational model. Journal of International Business Studies, Vol. 35, No. 5, pp. 350-370.

Luo, Yadong (2004). Building a Strong Foothold in an Emerging Market: A Link Between Resource Commitment and Environment Conditions. Journal of Management Studies, Vol. 41, No. 5, pp. 749-743.

Penfold, Michael (2002). "Los costos de la debilidad institucional". Debates IESA, Año 8, No. 1, Venezuela, Instituto de Estudios Superiores de Administración, pp. 12-19.

Prahalad, C. K. y Lieberthal, Kenneth (1998). The End of Corporate Imperialism. Harvard Business Review, Vol. 76, No. 4, EUA, Harvard University, pp. 68-79.

Roth, Kendall y Morrison, Allen J. (1992). Implementing global strategy: Characteristics of global subsidiary mandates. Journal of International Busi- ness Studies, Vol. 23, No. 4, pp. 715-735.

Sabal, Jaime (2002a). Los riesgos del riesgo país. Debates IESA, Año 7, No. 2-3, Venezuela, Instituto de Estudios Superiores de Administración, pp. 8185.

Sabal, Jaime (2002b). Financial decisions in emerging markets. Oxford University Press, New York.

Santos, Miguel y Villasmil, Ricardo (2006). La economía venezolana durante el último cuarto de siglo: análisis y propuestas para alcanzar el desarrollo. En Equipo Acuerdo Social: Venezuela: Un acuerdo para alcanzar el desarrollo, pp. 341-367. Publicaciones UCAB, Caracas.

Stake, R. E. (1995). The Art of Case Study Research. Sage Publications, Thousand Oaks.

UNCTAD (2009). World Investment Report 2009: Transnational Corporations, Agricultural Production and Development. United Nations, New York \& Geneva.

Weick, Karl E. (1996). Drop your tools: An allegory for organizational studies. Administrative Science Quarterly, Vol. 41, No. 2, pp. 301-313.

Wernerfelt, Birger (1984). A resource based view of the firm. Strategic Management Journal, Vol. 5, No. 2, pp. 171180.

White, Roderick E. y Poynter, Thomas A. (1984). Strategies for ForeignOwned Subsidiaries in Canada. Business Quarterly, Vol. 49, No. 2, pp. 59-69.

Yin, Robert K. (1994). Case Study Research: Design and Methods. $2^{\mathrm{a}} \mathrm{Ed}$. Sage Publications, Thousand Oaks. 